\title{
Phosphodiesterase 4 inhibition affects both the direct and indirect pathway: an electrophysiological study examining the tri-phasic response in the substantia nigra pars reticulata
}

\author{
P. R. A. Heckman ${ }^{1,2} \cdot$ J. V. Schweimer ${ }^{3} \cdot$ T. Sharp ${ }^{3} \cdot$ J. Prickaerts ${ }^{1} \cdot$ \\ A. Blokland ${ }^{2}$
}

Received: 28 March 2017/ Accepted: 12 September 2017/Published online: 18 September 2017

(c) The Author(s) 2017. This article is an open access publication

\begin{abstract}
Fronto-striatal circuits constitute the neurobiological basis of many neuropsychiatric disorders. Part of the intracellular signaling within these circuits, including its dopaminergic modulation, is regulated by the cyclic adenosine monophosphate (cAMP)/protein kinase A (PKA) signaling cascade. Based on the overall expression in human fronto-striatal circuitry, we tested the effects of a cAMP selective phosphodiesterase 4 (PDE4) inhibitor on the tri-phasic response in the dorsomedial substantia nigra pars reticulata $(\mathrm{SNr})$ upon stimulation of the infralimbic cortex in rats. Our results show for the first time that stimulation of the cognitive infralimbic cortex leads to a tri-phasic response in $\mathrm{SNr}$ neurons. In addition and in line with previous biochemical and behavioral studies, PDE4 inhibition by roflumilast affects the direct pathway as well as the indirect pathway of which the latter appears more sensitive than the former.
\end{abstract}

Keywords Phosphodiesterase inhibitor - Roflumilast . Substantia nigra pars reticulata $\cdot$ Fronto-striatal circuits

$\triangle$ P. R. A. Heckman

pim.heckman@maastrichtuniversity.nl

1 Department of Psychiatry and Neuropsychology, School for Mental Health and Neuroscience (MHeNS), Maastricht University, PO box 616, 6200 MD Maastricht, The Netherlands

2 Department of Neuropsychology and Psychopharmacology, Maastricht University, PO box 616, 6200 MD Maastricht, The Netherlands

3 Department of Pharmacology, University of Oxford, Mansfield Road, Oxford OX1 3QT, UK

\section{Introduction}

The fronto-striatal circuits are parallel organized circuits running from the frontal cortex, through the basal ganglia structures, to the thalamus from where they project back to the frontal cortex (Alexander and Crutcher 1990; Alexander et al. 1986). Therefore, the fronto-striatal circuits are also known as cortico-striatal-thalamic circuits. The frontostriatal circuits can be divided into three groups based on biological function, i.e., motor, associative/cognitive and limbic (Alexander et al. 1986). All circuits are characterized by their modulation by dopamine at the level of the striatum (Greengard 2001; Greengard et al. 1999; Svenningsson et al. 2004) and their division into three pathways within the basal ganglia, i.e., the hyperdirect pathway, the direct pathway and the indirect pathway. Altogether, the fronto-striatal circuits comprise a complex mechanism of action and functionality, which is abundantly described elsewhere (e.g., Calabresi et al. 2014; Gerfen and Surmeier 2011; Haber and Rauch 2010; Surmeier et al. 2011, 2007). In short, the fronto-striatal circuits start with glutamatergic projections into the striatum (caudate nucleus, putamen and nucleus accumbens (NAc)). Within the basal ganglia all projections are GABA ( $\gamma$-aminobutyric acid)-ergic except for output from the subthalamic nucleus (STN) which is glutamatergic. In the striatum each fronto-striatal circuit splits into a direct and an indirect pathway. Cortical glutamatergic activation of a striatal direct pathway stimulates the release of GABA, having an inhibitory effect in the globus pallidus pars interna (GPi) and substantia nigra pars reticulata ( $\mathrm{SNr}$ ). As the $\mathrm{GPi}$ and $\mathrm{SNr}$ inhibit the thalamus, which in turn stimulates the cortex, activation of the direct pathway causes disinhibition of the thalamus leading to increased excitatory output of the neural network, and thereby activation of behavior (either motor, cognitive or 
limbic). The indirect pathway has the opposite effect. Activation of the indirect pathway induces GABA release in the globus pallidus pars externa (GPe) which normally inhibits the release of GABA to the STN. The STN is thus disinhibited and increases stimulation of the GPi/SNr, which in turn inhibits the thalamic stimulation back to the cortex and results in inhibition of behavior. Additionally, the hyperdirect pathway consists of cortical glutamatergic projections to the STN, thereby completely circumventing the striatum. The disinhibition of the STN increases glutamatergic excitation of the output nuclei $(\mathrm{GPi} / \mathrm{SNr})$. Increased activation of the $\mathrm{GPi} / \mathrm{SNr}$ results in increased release of GABA from the GPi/SNr to the thalamus. This increased inhibition of the thalamus results in inhibition of the thalamic stimulation to the cortex. In the end, activation of the hyperdirect pathways results in inhibition of behavior. Without cortical stimulation the GPi and the $\mathrm{SNr}$ function like autonomous pacemakers, tonically inhibiting the thalamus, thereby preventing cortical stimulation, i.e., behavioral output.

In the striatum, dopaminergic neurotransmission is regulated by the cyclic adenosine monophosphate (cAMP)/ protein kinase A (PKA) signaling cascade targeting effectors like cAMP response element-binding protein (CREB) and Dopamine- and cAMP-Regulated PhosphoProtein MR $32 \mathrm{kDa}$ (DARPP-32) (Mayr and Montminy 2001; Svenningsson et al. 2004). Consequently, dopaminergic signaling is regulated by phosphodiesterases (PDEs), as cAMP is degraded by PDEs. Because of its substrate cAMP and based on the overall expression in human fronto-striatal circuitry (Lakics et al. 2010), the PDE4 family is of particular interest (for a review see Heckman et al. 2016). However, little is known regarding the distinct expression of PDE4 within the three individual basal ganglia pathways.

Therefore, in the current study we used an in vivo electrophysiology approach to investigate the three basal ganglia pathways. For this we measured extracellular neural activity in the substantia nigra pars reticulata $(\mathrm{SNr})$ during frontal cortex stimulation (Kolomiets et al. 2003). In particular, we were interested in the circuits involving cognitive function rather than fronto-striatal circuits involving motor or limbic functions (Deniau et al. 1996). In rats, these encompass the fronto-striatal circuits originating in the medial prefrontal cortex (mPFC; prelimbic and infralimbic cortices) and the orbitofrontal cortex. We focused on the circuit originating in the infralimbic cortex instead of the prelimbic cortex or orbitofrontal cortex, because sparse evidence confirms the existence of the three pathways (hyper, direct and indirect pathways) in this circuit. Also, the infralimbic cortex projections leave the basal ganglia mainly via the ventral pallidum (e.g., Deniau et al. 1994; Groenewegen et al. 1999) with less projections via the $\mathrm{SNr}$ (Vertes 2004). We hypothesize that stimulation of the infralimbic cortex may lead to a tri-phasic response in the SNr (Maurice et al. 1999), topographically and functionally associated with the ventral parts of the basal ganglia. The temporal and topographic sensitivity of this electrophysiological response combined could directly address the distinctive effects, if any, of PDE4 inhibition in the three basal ganglia pathways, and thus determine PDE4 function and PDE4 inhibitor applicability to specifically influence fronto-striatal cognitive function.

\section{Experimental procedures}

\section{Animals}

A total of 24 male Wistar rats (age 3 months, $260-380 \mathrm{~g}$, Charles River, Margate, Kent, UK) were used. Animals were housed collectively with ad libitum access to food and water. In the animal facility a normal 12-h light/dark cycle was maintained. In vivo electrophysiological experiments including drug administration were conducted during day time. All experiments were conducted at the University of Oxford at the University Department of Pharmacology in accordance with the Animals (Scientific Procedures) Act 1986 (UK) and were approved by a local Ethical Review Process at the University of Oxford.

\section{Electrophysiological recordings}

General anesthesia was induced with isoflurane (Isoflu, Abbott, Queenborough, Kent, UK) and maintained with urethane $(1.3-1.5 \mathrm{mg} / \mathrm{kg}$, ethyl carbamate, Sigma, Steinheim, Germany), supplemented with doses of ketamine (30 mg/kg, i.m.; Narketan, Vetoquinol, Buckingham, Buckinghamshire, UK) and xylazine $(10 \mathrm{mg} / \mathrm{kg}$, i.m.; Rompun, Bayer, Newbury, Berkshire, UK) whenever necessary. Subsequently, animals were placed in a stereotaxic frame (David Kopf Instruments, Tujunga, CA, USA). Surgery site was pretreated with bupivacaine for local anesthesia. Corneal dehydration was prevented by applications of Lacrilube eye gel (Allergan, Coolock, Dublin, Ireland). Body temperature was maintained at $37 \pm 0.5{ }^{\circ} \mathrm{C}$ using a homeothermic heating blanket (Harvard Apparatus, Kent, UK).

An electrocorticogram (ECoG) was recorded over the left motor cortex $(2.6 \mathrm{~mm}$ posterior, $2.0 \mathrm{~mm}$ lateral to bregma) to monitor the depth of anesthesia (see Schweimer et al. 2011 for details). Craniotomies were performed above the infralimbic cortex $(3.2 \mathrm{~mm}$ anterior, $0.6 \mathrm{~mm}$ lateral to bregma) for the stimulation electrode; and above the $\mathrm{SNr}$ (5.2 $\mathrm{mm}$ posterior, $2.0 \mathrm{~mm}$ lateral to bregma) for the recording electrode (Paxinos and Watson 2009). Saline 
was applied to the exposed brain surface to prevent dehydration.

Extracellular neuronal activity was monitored with a 10-25 M $\Omega$ glass microelectrode filled with $1.5 \%$ Neurobiotin (Vectorlabs, Burlingame, CA, USA) in $0.5 \mathrm{M} \mathrm{NaCl}$ (tip diameter 1-1.5 $\mu \mathrm{m}$ ). The microelectrode was lowered into the $\mathrm{SNr}$ with a single-axis in vivo micromanipulator (IVM) controlled via LINLAB software (Scientifica, Uckfield, UK). Electrode signals were alternating current (AC)coupled, amplified (1000x), and band-pass filtered $(0.3-5 \mathrm{kHz})$ using a Neurolog system (Digitimer, Welwyn Garden City, Hertfordshire, UK) and acquired on-line through a Micro1401 interface and Spike2 software (Cambridge Electronic Design, Cambridge, Cambridgeshire, UK). Mains noise at $50 \mathrm{~Hz}$ was eliminated ('Humbug' filter, Brown et al. 2002) for single unit and ECoG recordings.

\section{Electrical stimulation of the medial prefrontal cortex (infralimbic cortex)}

Electrical stimulation of the infralimbic cortex (anterior: 3.2; lateral: 0.6 to bregma; ventral: $-4.5 \mathrm{~mm}$ from the cortical surface), ipsilateral to the recording $\mathrm{SNr}$ site, was performed with a bipolar coaxial stainless steel electrode $(200 \mu \mathrm{m}$ tip diameter, $500 \mu \mathrm{m}$ shaft diameter, $500 \mu \mathrm{m}$ exposed inner and outer contact; NE-100 Harvard Apparatus, UK) positioned stereotaxically according to Paxinos and Watson (2009). Electrical stimuli were generated from a constant current isolated stimulator (WPI A360 stimulus isolator, Sarasota, Florida, USA) controlled via a Master-8 (A.M.P.I., Israel). Stimulation consisted of monopolar pulses of $0.6 \mathrm{~ms}$ width and $500 \mu \mathrm{A}$ intensity delivered at a frequency of $1.4 \mathrm{~Hz}$.

\section{Drugs}

All drugs were freshly prepared before each experiment. Roflumilast (kindly provided by Takeda, Konstanz, Germany) was administered i.v. in accumulating doses $(0.0025,0.005$ and $0.01 \mathrm{mg} / \mathrm{kg})$. This was done to establish a dose-response curve and maximizing the data per animal, and thereby reduce animal numbers. Roflumilast was dissolved in 10\% Kolliphor HS 15 (Sigma-Aldrich, Schnelldorf, Germany) and $90 \%$ isotonic saline $(0.9 \%$, Scheller et al. 2014) to make it suitable for i.v. administration.

\section{Extracellular single unit recordings of the $\mathrm{SNr}$ neurons, pharmacological treatment and experimental design}

Initially, the recording electrode was lowered to: anterior: $-5.2 \mathrm{~mm}$, lateral: $2.0 \mathrm{~mm}$ lateral to bregma; ventral:
$-7.0 \mathrm{~mm}$ from the cortical surface according to Paxinos and Watson (2009). For each experiment the dorsal border of the $\mathrm{SNr}$ was located by identifying dopaminergic neurons of the SNc. Criteria for dopaminergic SNc neurons were broad action potentials $(>1.1 \mathrm{~ms})$, and low to moderate frequency discharge $(2-10 \mathrm{~Hz})$ (Bunney et al. 1973; Ungless and Grace 2012). Ventral of the SNc layer of dopaminergic neurons, $\mathrm{SNr}$ neurons can be found. Electrophysiological characteristics of the $\mathrm{SNr}$ neuron were thin spikes (width $<0.55 \mathrm{~ms}$ ), high-frequency discharge $(>10 \mathrm{~Hz})$ without a decrease in the spike amplitude (Bunney et al. 1973; Deniau et al. 1978; Kolomiets et al. 2003). Once an $\mathrm{SNr}$ neuron was detected a stable baseline firing rate was established for $2 \mathrm{~min}$, followed by a 2-min stimulation period to check for the typical tri-phasic response of the $\mathrm{SNr}$ (excitation-inhibition-excitation). Presence of a tri-phasic response was also used as electrophysiological characteristic of the $\mathrm{SNr}$ neuron. The recording pattern was repeated if the neuron exhibited a triphasic response to determine stable baseline spike train parameters as well as a stable tri-phasic response; followed by drug injections (i.v.) at 4-min intervals ( 2 min stimulation off and $2 \mathrm{~min}$ stimulation on). This allowed for testing of the effects of different doses of roflumilast on baseline spike train parameters as well as the $\mathrm{SNr}$ tri-phasic response (see Table 1 ).

\section{Histochemistry}

Following pharmacological treatment, animals were overdosed with pentobarbitone, brains were removed and kept in 4\% PFA overnight, before being transferred to a $30 \%$ sucrose solution for cryoprotection. Coronal sections $(30 \mu \mathrm{m})$ were cut using a cryostat (Bright Instruments Ltd, Luton, Bedfordshire, UK). Posterior sections containing the complete $\mathrm{SNr}$ were checked using a light-microscope (Leica Dialux 20) to verify recording electrode location. Additionally, PFC sections were stained using a standard Nissl-staining protocol to verify stimulation electrode location. Prior to the Nissl-staining, sections were mounted and hydrated in decreasing concentrations of ethanol. Following the Nissl-staining, sections were first dehydrated in increasing concentrations of ethanol and subsequently treated with isopropanol and xylene. Sections were examined under a light-microscope (Leica Dialux 20). Brightness and contrast of the images were adjusted using ImageJ software (version 1.50, ImageJ, National Institute of Health, Bethesda, MD, USA).

\section{Data and statistical analysis}

For the baseline periods, the interspike interval, coefficient of variation of the interspike interval, firing rate, and spike 
Table 1 Schematic overview of the experimental stimulation and recordings

\begin{tabular}{|c|c|c|}
\hline $\begin{array}{l}\text { Time }(\min ) \\
(T)\end{array}$ & $\begin{array}{l}\text { Stimulation infralimbic cortex } \\
\text { (on/off) }\end{array}$ & Experimental step \\
\hline 0 & Off & Detection $\mathrm{SNr}$ neuron according to electrophysiological characteristics \\
\hline 2 & Off & Establishment of stable baseline firing \\
\hline 4 & On & $\begin{array}{l}\text { Verification of typical tri-phasic response of the } \mathrm{SNr} \text { (excitation-inhibition-excitation) after } \\
\text { stimulation infralimbic cortex }\end{array}$ \\
\hline 6 & Off & Verification stable baseline spike train parameters \\
\hline 8 & On & Verification stable tri-phasic response \\
\hline 10 & Off & Injection vehicle (i.v.) \\
\hline 10 & Off & Measuring effects dosing on baseline spike train parameters \\
\hline 12 & On & Measuring effects dosing on tri-phasic response \\
\hline 14 & Off & Injection $0.0025 \mathrm{mg} / \mathrm{kg}$ roflumilast (i.v.) \\
\hline 14 & Off & Measuring effects dosing on baseline spike train parameters \\
\hline 16 & On & Measuring effects dosing on tri-phasic response \\
\hline 18 & Off & Injection $0.005 \mathrm{mg} / \mathrm{kg}$ roflumilast (i.v.) \\
\hline 18 & Off & Measuring effects dosing on baseline spike train parameters \\
\hline 20 & On & Measuring effects dosing on tri-phasic response \\
\hline 22 & Off & Injection $0.01 \mathrm{mg} / \mathrm{kg}$ roflumilast (i.v.) \\
\hline 22 & Off & Measuring effects dosing on baseline spike train parameters \\
\hline 24 & On & Measuring effects dosing on tri-phasic response \\
\hline 26 & Off & Juxtacellular labeling or iontophoresis \\
\hline 86 & Off & Leave to settle \\
\hline 131 & Off & Transcardial perfusion \\
\hline
\end{tabular}

SNr substantia nigra pars reticulata, i.v. intravenous injection

waveform width (time taken from a 5\% increase from baseline to the first trough) were calculated for all neurons (e.g., Brouard et al. 2015; Schweimer et al. 2011).

We calculated the peristimulus-time histograms (PSTH) and response magnitudes as previously described in Beyeler et al. (2010). PSTH were generated for every 2-min stimulation period (5-ms bins). The baseline was defined as the mean of the spikes of the $100 \mathrm{~ms}$ epoch in each, individual PSTH preceding the cortical stimulation. In addition, the standard deviation (SD) of the baseline was determined. A significant excitation was considered if the mean of all bins within the excitatory epoch exceeded the mean baseline activity by 2 SD. An inhibition was defined as a period during which the mean of bins of the inhibitory epoch is below $70 \%$ of the baseline mean (Georges and Aston-Jones 2002).

Excitatory response magnitudes $\left(R_{\text {mags }}\right)$ were normalized for different levels of baseline activity, allowing for comparison of drug effects on evoked responses independent of effects on baseline activity or differences in firing rate (Georges and Aston-Jones 2002). $R_{\text {mags }}$ for excitation were calculated with the following equation: Excitation $R_{\text {mag }}=$ (counts in excitatory epoch) - (mean counts per baseline bin $\times$ number of bins in excitatory epoch). In contrast, for the inhibition the $R_{\mathrm{mag}}$ is expressed in absolute counts due to the low number of counts in the inhibition period. Expressing these low counts in the same way as positive $R_{\text {mags }}$ induces artificially high values (i.e., percentages) and high variability. The count numbers during the inhibition period were normally distributed and were comparable between the neurons. Finally, to determine the latency and the duration of each phase of the response, PSTH were generated from " 2 min of" trials using 1-ms bins (Beyeler et al. 2010).

Statistical analysis was carried out using IBM SPSS Statistics 24 software (IBM, Portsmouth, UK). The raw data were checked for outliers and normality of distribution. Missing values were replaced using the multiple imputation function in SPSS using 5 imputations (Olivier et al. 2009). One-way repeated measures analysis of variance (ANOVA) followed by Sidak's post hoc test was used. Data are depicted as mean \pm SEM.

\section{Results}

\section{Effects of roflumilast on basal $\mathrm{SNr}$ neuron activity}

To determine the effect of roflumilast in the cognitive fronto-striatal circuit originating in the infralimbic cortex 
we recorded in 24 rats 65 neurons that exhibited an electrophysiological profile corresponding to the $\mathrm{SNr}$ criteria (see 'Experimental procedures' section). Figure 1 shows a typical tri-phasic response of the $\mathrm{SNr}$ after infralimbic cortex stimulation including baseline firing properties. Recordings of these neurons showed that about $50 \%$ of these neurons $(n=33)$ responded to electrical stimulation of the infralimbic cortex (infralimbic cortex electrode location verified; see Fig. 2). Among these 33 neurons only $18 \%$ showed a tri-phasic response $(n=6)$. This percentage corroborates earlier findings observed in earlier studies where the auditory-, motor-, and pre-limbic cortices were stimulated (Kolomiets et al. 2003; Maurice et al. 1999). Only $\mathrm{SNr}$ neurons exhibiting a tri-phasic response were included in the analysis because they represent activation of the full circuit of interest (see Fig. 2).

Before analyzing the effects of roflumilast on any of the baseline spike train parameters or the tri-phasic response we first verified that the (repeated) stimulation of the infralimbic cortex or the injection had no effect on any of the parameters investigated. Neither stimulation itself nor the injection had an effect on the baseline firing (data not shown). Subsequently, roflumilast had no effect on baseline firing properties of the recorded neurons (firing rate, or regularity (COV IS), data not shown).

The physiological response to stimulation of the $\mathrm{SNr}$ neurons was compatible with previous data (Beyeler et al. 2010; Kolomiets et al. 2003; Ryan and Sanders 1994) regarding the delay of appearance and the duration of each phase of the tri-phasic response. Location of responding (cognitive) neurons within the $\mathrm{SNr}$ is in line with the division of motor, cognitive (associative) and limbic areas within the SNr (e.g., Kolomiets et al. 2003).

\section{Effects of roflumilast on mPFC stimulation-evoked responses of $\mathrm{SNr}$ neurons}

The tri-phasic response that was measured represents activation of the hyper direct, direct and indirect basal ganglia pathways. Each individual pathway is measured as early excitation, inhibition and late excitation, respectively. At stimulation (no treatment), latencies and durations were as follow (in ms \pm SEM; $n=6$ ) for the early excitation phase: $4.8 \pm 1.4$ and $10.8 \pm 1.5$, respectively. For the inhibition phase this was $20.17 \pm 1.8$ and $12.67 \pm 0.99$, respectively, and for the late excitation phase these values were $36.5 \pm 2.5$ and $13.67 \pm 2.0$, respectively. These values were not affected during the different stages of the experiment and did not change after roflumilast treatment. Thus, roflumilast had no effect on the latency or duration of the tri-phasic response.

No effects of roflumilast were observed for the $R_{\text {mag }}$ of the early excitation phase (Fig. 3a). Roflumilast increased the firing rate during the inhibition phase $(F(3,15)=6.294, p=0.006)$. Post hoc test revealed a $117 \%$ increase in the firing rate after $0.01 \mathrm{mg} / \mathrm{kg}$ (Fig. 3b; $p=0.049)$. The calculated effect size for this difference
Fig. 1 Effects of roflumilast on the tri-phasic response. Raster plot and peristimulus-time histogram demonstrating triphasic (excitation-inhibitionexcitation) response of a $\mathrm{SNr}$ neuron to infralimbic cortex stimulation. Vertical line at $T=0$ represents the time of stimulation; E1 = early excitation phase of the tri-phasic response; I = inhibition phase of the tri-phasic response; $\mathrm{E} 2$ = late excitation phase of the tri-phasic response. Baseline firing properties of $\mathrm{SNr}$ neurons displaying a tri-phasic response $(N=6)$ : Average spike waveform width: $0.0013 \pm 0.00021$ (s); Firing rate $(\mathrm{Hz}): 25.89 \pm 3.81$; Coefficient of variation of the interspike interval: $0.25 \pm 0.017$; Latency first excitation(s): $0.0048 \pm 0.0014$
Sweeps
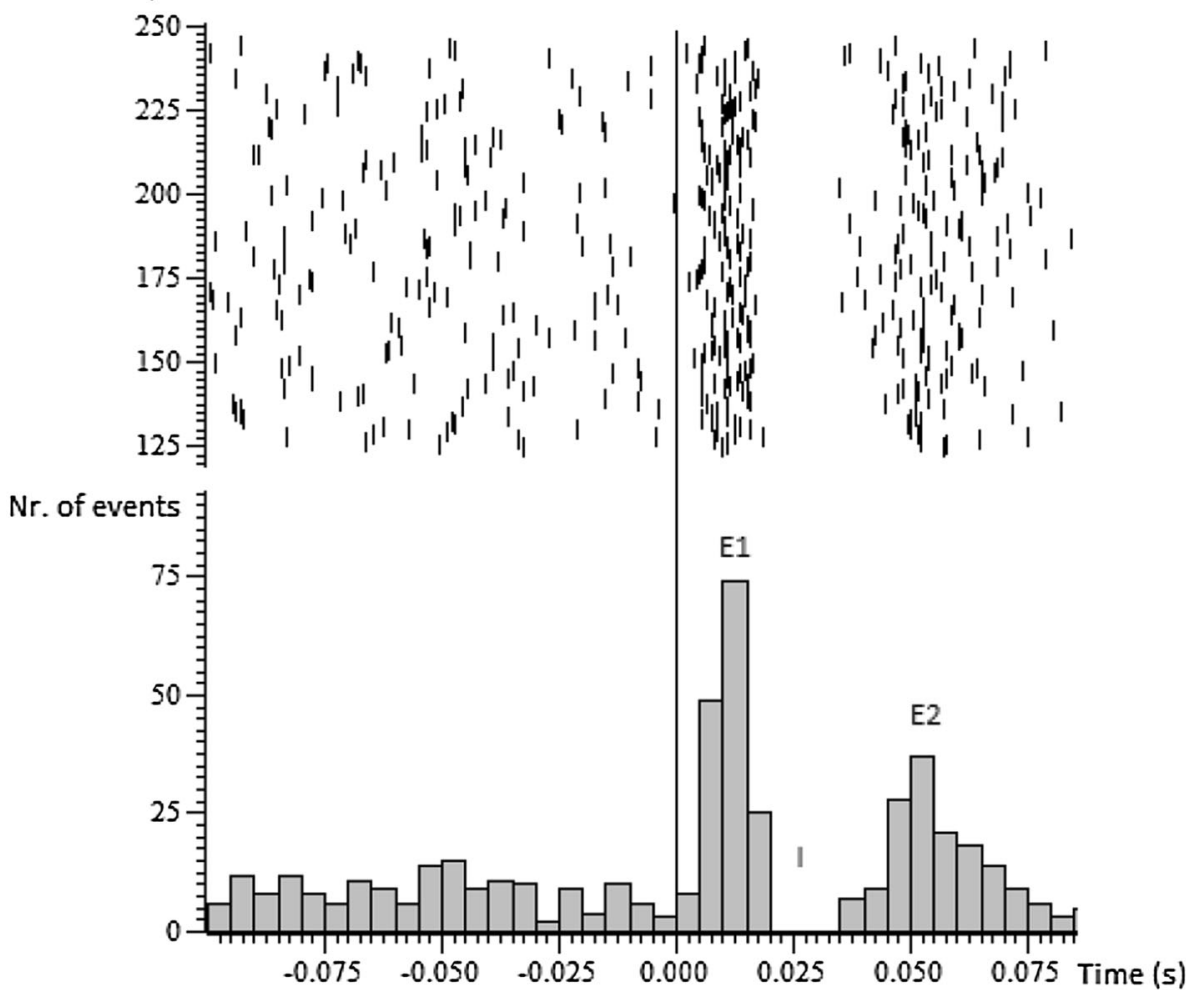

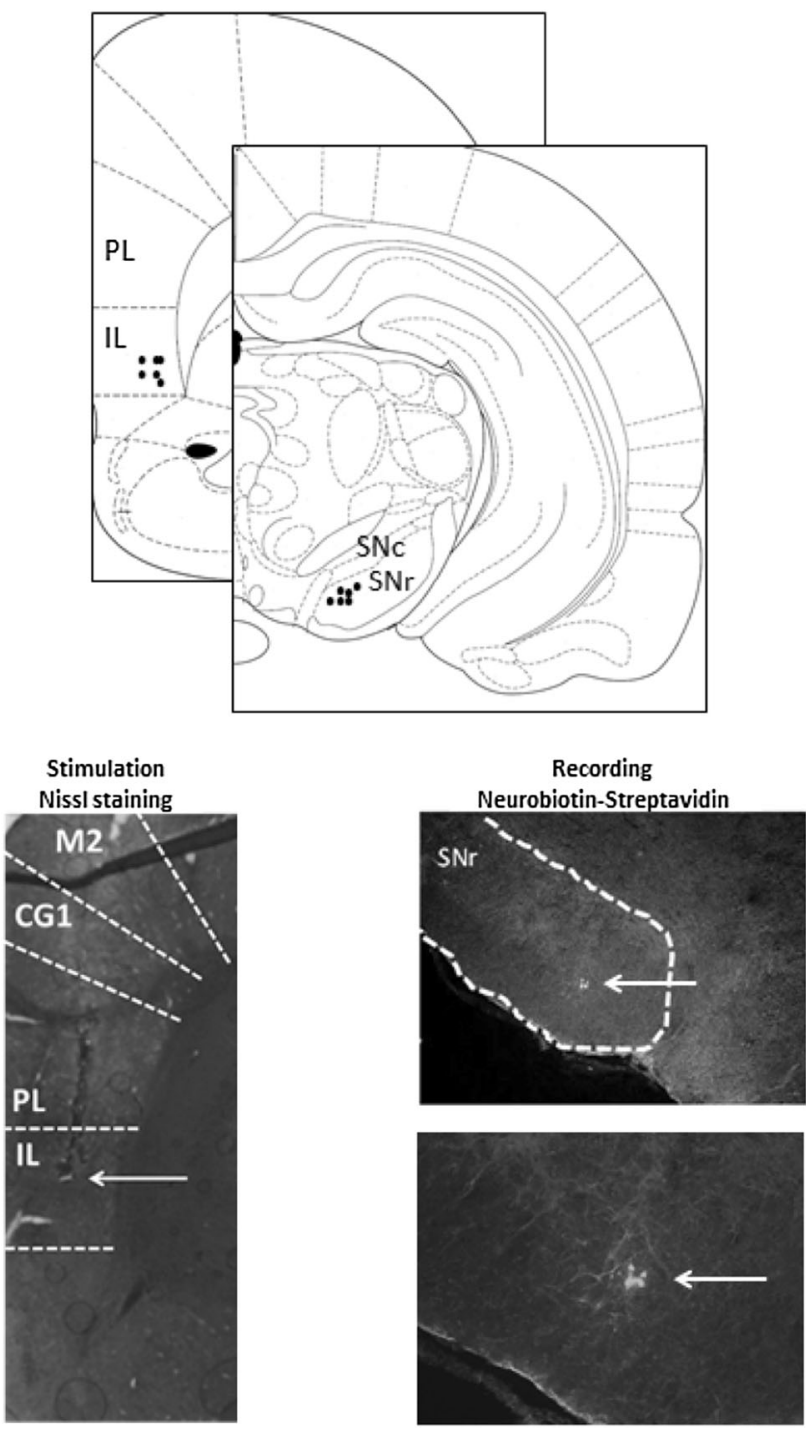

Fig. 2 Upper section: Graphical depiction of stimulation and recording electrode locations for the 6 neurons exhibiting a tri-phasic response (stimulation electrode: coronal section anterior: $3.2 \mathrm{~mm}$ to bregma; recording electrode: coronal section anterior: $-5.3 \mathrm{~mm}$ to bregma). Lower section: On the left hand side, the medial prefrontal cortex is shown including the large shaft created by the stimulation electrode. The shaft extends from the dorsal surface of the brain ventrally into the IL (Nissl-staining). On the right hand side, 2 figures are shown depicting the location of the tip of the recording electrode in the $\mathrm{SNr}$ via iontophoresis (neurobiotin-streptavidin staining). The lower image is a magnification of the upper image. CG1 = cingulate cortex area $1 ; \mathrm{M} 2=$ secondary motor cortex; $\mathrm{PL}=$ prelimbic cortex $; \mathrm{IL}=$ infralimbic cortex; $\mathrm{SNc}=$ substantia nigra pars compacta (dorsal tier); $\mathrm{SNr}=$ substantia nigra pars reticulata; arrows indicate electrode tip locations

was 1.06 (Cohen's D), which can be considered as a large effect size. For the late excitation phase the $R_{\mathrm{mag}}$ the repeated measures ANOVA showed a marginal treatment effect $(F(3,15)=3.05, p=0.06)$. However, post hoc analysis revealed a $50 \%$ decrease in $R_{\text {mag }}$ after $0.005 \mathrm{mg} /$ $\mathrm{kg}$ roflumilast treatment when compared with vehicle
A Early excitation phase (E1)
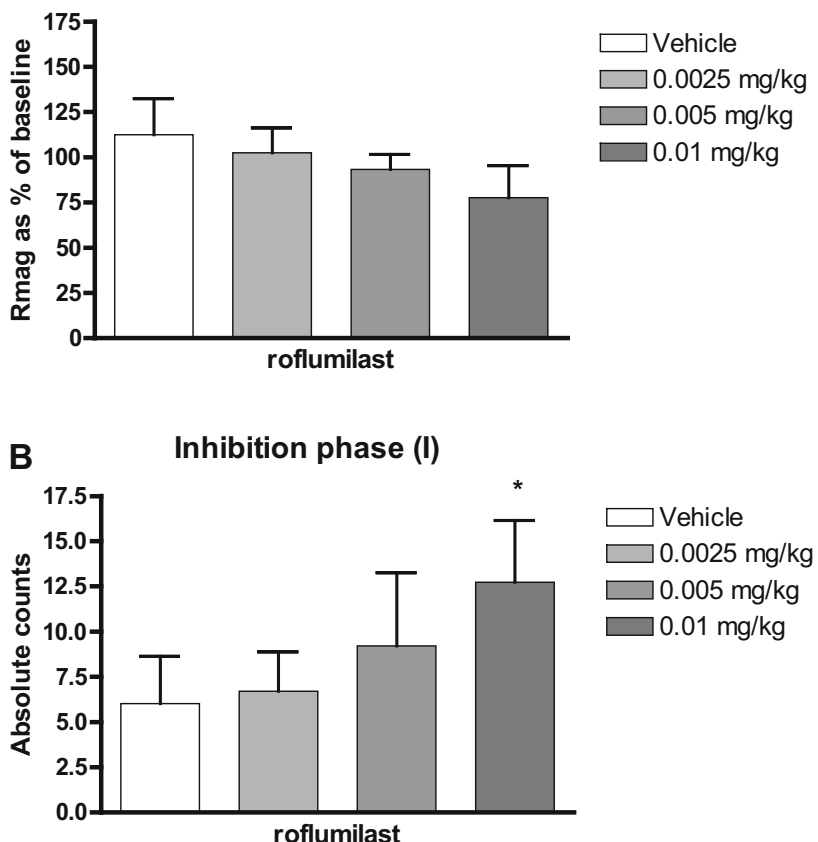

C Late excitation phase (E2)
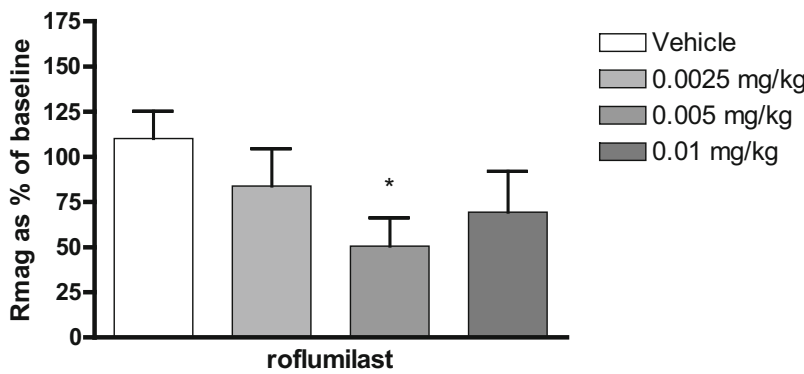
$0.01 \mathrm{mg} / \mathrm{kg}$

Fig. 3 Effects of the PDE4 inhibitor roflumilast on the magnitude of the electrophysiological response of the $\mathrm{SNr}$ neurons to electrical stimulation of the infralimbic cortex. Each bar represents the magnitude of the response ( $R_{\mathrm{mag}}$ as \% of baseline for the early (E1) and late (E2) excitation phases, and absolute counts for the inhibition (I) phase). a Roflumilast did not affect the $R_{\text {mag }}$ of the early excitation phase. b Roflumilast decreased the magnitude of the inhibition as depicted by an increase in the absolute number of counts at the $0.01 \mathrm{mg} / \mathrm{kg}$ dose. $\mathbf{c}$ In the late excitation phase a decrease in $R_{\text {mag }}$ was found at the $0.005 \mathrm{mg} / \mathrm{kg}$ dose

(Fig. 3c; $p=0.035$ ). The calculated effect size for this difference was 1.58 (Cohen's D), which can be considered as a large effect size.

\section{Discussion}

The current study was conducted to address the potentially distinctive effects of PDE4 inhibition on the three basal ganglia pathways: the hyperdirect, direct and indirect pathway. This was done by examining the effects on the 
tri-phasic (excitation-inhibition-excitation) response of the $\mathrm{SNr}$ after infralimbic cortex stimulation. An increase or decrease in the number of action potentials, as represented by the $\mathrm{R}_{\text {mag }}$ or absolute numbers, within a particular phase of the tri-phasic response, is indicative of an effect of roflumilast in the pathway represented by that particular phase of the tri-phasic response. Our results provide support for the hypothesis that PDE4 inhibition affects the direct and indirect pathways of the basal ganglia, but not the hyperdirect pathway. This conclusion is based on the fact that roflumilast only affected the second and third phases of the tri-phasic response, representing the direct and indirect pathways, respectively. Roflumilast did not change the $\mathrm{R}_{\text {mag }}$ in the first phase of the tri-phasic response, representing the hyperdirect pathway. Therefore, we conclude that roflumilast does not exert effects in the hyperdirect pathway. Since the hyperdirect pathway is also part of the indirect pathway, the effects of roflumilast in the indirect pathway observed during our studies, are most likely not exerted in the trajectory of STN-SNr/GPi-thalamus. This conclusion is in line with previous findings supporting a role for PDE4 in the direct and indirect pathways originating in striatal medium spiny neurons (Heckman et al. 2016; Nishi et al. 2008, 2011; Nishi and Snyder 2010). The current data support the notion that PDE4 has a preferential role in the indirect pathway since PDE4 inhibition reduced the $\mathrm{R}_{\text {mag }}$ of the indirect pathway at a lower dose when compared to the neuronal activity in the direct pathway.

Previous studies showed that PDE4 predominantly regulates cAMP/PKA signaling at striatal dopaminergic terminals and associated dopamine synthesis and release (Nishi et al. 2008; Schoffelmeer et al. 1985; Yamashita et al. 1997a, b). Secondary to this, PDE4 regulates cAMP/PKA signaling in medium spiny neurons. This is the case for both the striatopallidal as well as the striatonigral pathway as was observed by increased levels of phospho-DARPP-32 in neostriatal slices in both pathways after PDE4 inhibitor treatment (Nishi et al. 2008). These findings were supported by immunohistochemistry data. Of note, higher expression levels were observed in (indirect) striatopallidal neurons but only the PDE4B subtype was examined. Interestingly, PDE4 inhibition only potentiated adenosine $A_{2 a}$ receptor -induced phosphorylation of DARPP-32 by the adenosine $\mathrm{A}_{2 \mathrm{a}}$ receptor agonist CGS21680, whereas no additional effects of PDE4 inhibition were observed after stimulation of the dopamine D1 receptor with SKF81297 (Nishi and Snyder 2010). The latter can be interpreted as a preferential effect of PDE4 inhibition in striatopallidal neurons as $\mathrm{A}_{2 \mathrm{a}}$ receptors are located on indirect pathway medium spiny neurons and D1 receptors on direct pathway medium spiny neurons (Gerfen and Surmeier 2011).

However, slices do not need to represent the in vivo situation as for instance, as mentioned earlier, the observed increase in DARPP-32 phosphorylation after rolipram treatment in neostriatal slices could not be observed in vivo by the same group (Nishi et al. 2008). Clearly, when studying the fronto-striatal circuitry the striatal slices can only model part of this circuitry and may therefore not be the most suitable model for studying a fully intact functional circuit. Therefore, the current study is unique in providing support for a more sensitive indirect pathway for PDE4 inhibition.

The more potent effect of roflumilast in the indirect pathway can be hypothesized to occur from several possibilities. One option would entail lower levels of PDE4 expression in medium spiny neurons of the indirect striatopallidal pathway compared to the direct striatonigral pathway (Niccolini et al. 2017). This way, the same dose of the compound can induce larger effects since absolute lower number of PDE4 proteins in the indirect pathway compared to the direct pathway. Another possibility to explain the observed effect would be that the direct pathway contains higher levels of PDE4 when compared to the indirect pathway. That way a higher dose of roflumilast is needed to inhibit higher levels of PDE4 compared to the indirect pathway before cAMP and related signaling is affected.

Also, the tri-phasic response measured in the $\mathrm{SNr}$ originates in the frontal cortex and is modulated at many different levels throughout the system before reaching the output module of the basal ganglia. For instance, even though the same intracellular effectors are involved in frontal and striatal dopaminergic signaling, biosensor imaging in mouse brain slice preparations showed profound differences in the D1 response between pyramidal cortical neurons and striatal medium spiny neurons (Castro et al. 2013). Also, effects in other cognitive, motor or limbic fronto-striatal circuits could affect the currently investigated infralimbic circuit, including projections coming from cingulate cortex, orbital cortex, parietal cortex and temporal cortex (Schmitt et al. 2016). Furthermore, it is unknown in which nigral and pallidal areas or where within the subthalamic nucleus PDE4 is expressed (e.g., Niccolini et al. 2017). The abundant number of feedback and feedforward connections within the circuits as well as their mediation and modulation by several neurotransmitter systems add to the complexity of predicting the net effect reaching the output modules of the basal ganglia (Calabresi et al. 2014; Gerfen and Surmeier 2011; Schmitt et al. 2016; Surmeier et al. 2011). The latter aspects could be responsible for the unexpected finding in both pathways, i.e., increased firing in the inhibition phase and decreased firing in the late excitation phase after roflumilast treatment. Future studies will have to investigate these possible mechanisms at the cellular and molecular level further (e.g., Nishi et al. 2017; Nishi and Shuto 2017). 
The functional implications of current results entail a more promising role for PDE4 inhibitors, or at least roflumilast, in disorders benefiting from less activation of the indirect pathway, i.e., less inhibition to cortical areas. This will add to the, in the current study neglected, main effect of roflumilast at dopaminergic terminals, i.e., dopamine release. Binding of dopamine to D1 and D2 receptors on striatonigral and striatopallidal medium spiny neurons will activate the direct pathway and inhibit the indirect pathway, respectively. Together, this can be expected to greatly benefit disorders characterized by hypodopaminergia, including attention deficit hyperactivity disorder (ADHD) and Parkinson's disease.

Even though our results are in line with previous findings in neostriatal slices and on behavioral outcomes, some difference must be highlighted. For instance, in the current study we examined the effects of a PDE4 inhibitor in anesthetized animals while behavioral studies discussed above examine fully awake and active animals. This means that, although the brain circuitry is intact and functional, it cannot be ruled out that different effects of roflumilast could be observed when conducting electrophysiological recordings in fully awake and freely moving animals. Since PDE4 is most prominently expressed at dopaminergic terminals affecting synthesis and release of dopamine in the striatum, a dopaminergic challenge of the brain circuitry may greatly affect fronto-striatal signaling. The latter is more likely to occur in freely moving animals during behavioral tasks compared to anesthetized animals (Sabeti et al. 2003). However, the former would also introduce more noise and thus variability in the data, when the research question reflects merely the examination of the effects of the drug. Another difference to address is that in the study examining striatal slices, the classic PDE4 inhibitor rolipram was used opposed to roflumilast in the current study, even though both inhibitors are selective for PDE4 over other PDE subfamilies and both have equal selectivity for the four PDE4 subtypes (Hatzelmann et al. 2010; Krause and Kuhne 1988).

In line with previous studies (e.g., Deniau et al. 1994; Groenewegen et al. 1999; Vertes 2004) cells exhibiting a triphasic response were located in the dorsomedial $\mathrm{SNr}$. Results of the current study further add to the evidence that cognitive projections, or at least those originating in the infralimbic cortex, can induce a tri-phasic response in the SNr. As a result, it also implies the existence of a division within the basal ganglia into a hyper, direct and indirect pathway, in the cognitive fronto-striatal circuit originating in the infralimbic cortex. Thus, this also confirms the hypothesis of the existence of the three pathways in the cognitive fronto-striatal circuits as they do in the motor or limbic circuits (e.g., Beyeler et al. 2010; Maurice et al. 1999).
In conclusion, our results show that stimulation of the infralimbic cortex leads to a tri-phasic response in a subset of neurons in the SNr. This is topographically and functionally associated with the cognitive parts of the basal ganglia, that can be used to investigate distinctive effects of drugs within the basal ganglia circuitry. The temporal and topographic sensitivity of this tri-phasic electrophysiological response combined with the neuroanatomical markers directly addressed the distinctive effects of PDE4 inhibition by roflumilast in the three basal ganglia pathways of the cognitive fronto-striatal circuitry. PDE4 inhibition by roflumilast appears to affect both the direct pathway as well as the indirect pathway with a relative preference for the latter. These findings are in line with previous in vitro and in vivo studies. Further studies may reveal whether PDE4 inhibition could be considered as a possible treatment for cognitive deficits related to fronto-striatal disorders in which either the direct or indirect pathway is affected (e.g., schizophrenia and ADHD, Huntington's disease and Parkinson's disease).

Acknowledgements PRAH is financially supported by the Human Enhancement and Learning (HEaL) initiative of Maastricht University. The current project has been supported by the medical Foundation "De Drie Lichten" in The Netherlands. We like to thank Prof. Dr. Peter Magill (Oxford) for his expert advice regarding basal ganglia electrophysiological recordings. We also like to thank Dr. Grant Churchill (Oxford) for his help finding a suitable vehicle and dissolving roflumilast.

\section{Compliance with ethical standards}

Conflict of interest $\mathrm{AB}$ and JP have a proprietary interest in the PDE4 inhibitor roflumilast.

Ethical approval All applicable international, national, and/or institutional guidelines for the care and use of animals were followed.

Human and animal rights All experiments were conducted at the University of Oxford at the University Department of Pharmacology in accordance with the Animals (Scientific Procedures) Act 1986 (UK) and were approved by a local Ethical Review Process at the University of Oxford.

Open Access This article is distributed under the terms of the Creative Commons Attribution 4.0 International License (http://crea tivecommons.org/licenses/by/4.0/), which permits unrestricted use, distribution, and reproduction in any medium, provided you give appropriate credit to the original author(s) and the source, provide a link to the Creative Commons license, and indicate if changes were made.

\section{References}

Alexander GE, Crutcher MD (1990) Functional architecture of basal ganglia circuits: neural substrates of parallel processing. Trends Neurosci 13:266-271 
Alexander GE, DeLong MR, Strick PL (1986) Parallel organization of functionally segregated circuits linking basal ganglia and cortex. Annu Rev Neurosci 9:357-381

Beyeler A et al (2010) Stimulation of serotonin2C receptors elicits abnormal oral movements by acting on pathways other than the sensorimotor one in the rat basal ganglia. Neuroscience 169:158-170. doi:10.1016/j.neuroscience.2010.04.061

Brouard JT, Schweimer JV, Houlton R, Burnham KE, Queree P, Sharp T (2015) Pharmacological evidence for 5-HT6 receptor modulation of 5-HT neuron firing in vivo. ACS Chem Neurosci 6:1241-1247. doi:10.1021/acschemneuro.5b00061

Brown P, Kupsch A, Magill PJ, Sharott A, Harnack D, Meissner W (2002) Oscillatory local field potentials recorded from the subthalamic nucleus of the alert rat. Exp Neurol 177:581-585

Bunney BS, Walters JR, Roth RH, Aghajanian GK (1973) Dopaminergic neurons: effect of antipsychotic drugs and amphetamine on single cell activity. J Pharmacol Exp Ther 185:560-571

Calabresi P, Picconi B, Tozzi A, Ghiglieri V, Di Filippo M (2014) Direct and indirect pathways of basal ganglia: a critical reappraisal. Nat Neurosci 17:1022-1030. doi:10.1038/nn.3743

Castro LR et al (2013) Striatal neurones have a specific ability to respond to phasic dopamine release. J Physiol 591:3197-3214. doi:10.1113/jphysiol.2013.252197

Deniau JM, Hammond C, Riszk A, Feger J (1978) Electrophysiological properties of identified output neurons of the rat substantia nigra (pars compacta and pars reticulata): evidences for the existence of branched neurons. Exp Brain Res 32:409-422

Deniau JM, Menetrey A, Thierry AM (1994) Indirect nucleus accumbens input to the prefrontal cortex via the substantia nigra pars reticulata: a combined anatomical and electrophysiological study in the rat. Neuroscience 61:533-545

Deniau JM, Menetrey A, Charpier S (1996) The lamellar organization of the rat substantia nigra pars reticulata: segregated patterns of striatal afferents and relationship to the topography of corticostriatal projections. Neuroscience 73:761-781

Georges F, Aston-Jones G (2002) Activation of ventral tegmental area cells by the bed nucleus of the stria terminalis: a novel excitatory amino acid input to midbrain dopamine neurons. J Neurosci 22:5173-5187

Gerfen CR, Surmeier DJ (2011) Modulation of striatal projection systems by dopamine. Annu Rev Neurosci 34:441-466. doi:10. 1146/annurev-neuro-061010-113641

Greengard P (2001) The neurobiology of dopamine signaling. Biosci Rep 21:247-269

Greengard P, Allen PB, Nairn AC (1999) Beyond the dopamine receptor: the DARPP-32/protein phosphatase-1 cascade. Neuron 23:435-447

Groenewegen HJ, Galis-de Graaf Y, Smeets WJ (1999) Integration and segregation of limbic cortico-striatal loops at the thalamic level: an experimental tracing study in rats. J Chem Neuroanat 16:167-185

Haber SN, Rauch SL (2010) Neurocircuitry: a window into the networks underlying neuropsychiatric disease. Neuropsychopharmacology 35:1-3. doi:10.1038/npp.2009.146

Hatzelmann A et al (2010) The preclinical pharmacology of roflumilast - a selective, oral phosphodiesterase 4 inhibitor in development for chronic obstructive pulmonary disease. Pulm Pharmacol Ther 23:235-256. doi:10.1016/j.pupt.2010.03.011

Heckman PR, van Duinen MA, Bollen EP, Nishi A, Wennogle L, Blokland A, Prickaerts J (2016) Phosphodiesterase inhibition and regulation of dopaminergic frontal and striatal functioning: clinical implications. Int J Neuropsychopharmacol. doi:10.1093/ ijnp/pyw030

Kolomiets BP, Deniau JM, Glowinski J, Thierry AM (2003) Basal ganglia and processing of cortical information: functional interactions between trans-striatal and trans-subthalamic circuits in the substantia nigra pars reticulata. Neuroscience 117:931-938

Krause W, Kuhne G (1988) Pharmacokinetics of rolipram in the rhesus and cynomolgus monkeys, the rat and the rabbit. Studies on species differences. Xenobiotica 18:561-571. doi:10.3109/ 00498258809041693

Lakics V, Karran EH, Boess FG (2010) Quantitative comparison of phosphodiesterase mRNA distribution in human brain and peripheral tissues. Neuropharmacology 59:367-374. doi:10. 1016/j.neuropharm.2010.05.004

Maurice N, Deniau JM, Glowinski J, Thierry AM (1999) Relationships between the prefrontal cortex and the basal ganglia in the rat: physiology of the cortico-nigral circuits. J Neurosci 19:4674-4681

Mayr B, Montminy M (2001) Transcriptional regulation by the phosphorylation-dependent factor CREB. Nat Rev Mol Cell Biol 2:599-609. doi: $10.1038 / 35085068$

Niccolini F et al (2017) Loss of phosphodiesterase 4 in Parkinson disease: relevance to cognitive deficits. Neurology. doi:10.1212/ wnl.0000000000004201

Nishi A, Shuto T (2017) Potential for targeting dopamine/DARPP-32 signaling in neuropsychiatric and neurodegenerative disorders. Expert Opin Ther Targets. doi:10.1080/14728222.2017.1279149

Nishi A, Snyder GL (2010) Advanced research on dopamine signaling to develop drugs for the treatment of mental disorders: biochemical and behavioral profiles of phosphodiesterase inhibition in dopaminergic neurotransmission. J Pharmacol Sci 114:6-16

Nishi A et al (2008) Distinct roles of PDE4 and PDE10A in the regulation of cAMP/PKA signaling in the striatum. J Neurosci 28:10460-10471. doi:10.1523/jneurosci.2518-08.2008

Nishi A, Kuroiwa M, Shuto T (2011) Mechanisms for the modulation of dopamine $\mathrm{d}(1)$ receptor signaling in striatal neurons. Front Neuroanat 5:43. doi:10.3389/fnana.2011.00043

Nishi A et al (2017) Glutamate counteracts dopamine/PKA signaling via dephosphorylation of DARPP-32 Ser-97 and alteration of its cytonuclear distribution. J Biol Chem 292:1462-1476. doi:10. 1074/jbc.M116.752402

Olivier JD, Jans LA, Blokland A, Broers NJ, Homberg JR, Ellenbroek BA, Cools AR (2009) Serotonin transporter deficiency in rats contributes to impaired object memory. Genes, brain, and behavior 8:829-834. doi:10.1111/j.1601-183X.2009.00530.x

Paxinos G, Watson C, Carrive P, Kirkcaldie M, Ashwell K (2009) Chemoarchitectonic atlas of the rat brain. 2nd edn. Elsevier Academic Press, San Diego

Ryan LJ, Sanders DJ (1994) Subthalamic nucleus and globus pallidus lesions alter activity in nigrothalamic neurons in rats. Brain Res Bull 34:19-26

Sabeti J, Gerhardt GA, Zahniser NR (2003) Chloral hydrate and ethanol, but not urethane, alter the clearance of exogenous dopamine recorded by chronoamperometry in striatum of unrestrained rats. Neurosci Lett 343:9-12

Scheller KJ, Williams SJ, Lawrence AJ, Jarrott B, Djouma E (2014) An improved method to prepare an injectable microemulsion of the galanin-receptor 3 selective antagonist, SNAP 37889, using Kolliphor((R)) HS 15. MethodsX 1:212-216. doi:10.1016/j.mex. 2014.09.003

Schmitt O, Eipert P, Kettlitz R, Lessmann F, Wree A (2016) The connectome of the basal ganglia. Brain Struct Function 221:753-814. doi:10.1007/s00429-014-0936-0

Schoffelmeer AN, Wardeh G, Mulder AH (1985) Cyclic AMP facilitates the electrically evoked release of radiolabelled noradrenaline, dopamine and 5-hydroxytryptamine from rat brain slices. Naunyn Schmiedebergs Arch Pharmacol 330:74-76

Schweimer JV, Mallet N, Sharp T, Ungless MA (2011) Spike-timing relationship of neurochemically-identified dorsal raphe neurons 
during cortical slow oscillations. Neuroscience 196:115-123. doi:10.1016/j.neuroscience.2011.08.072

Surmeier DJ, Ding J, Day M, Wang Z, Shen W (2007) D1 and D2 dopamine-receptor modulation of striatal glutamatergic signaling in striatal medium spiny neurons. Trends Neurosci 30:228-235. doi:10.1016/j.tins.2007.03.008

Surmeier DJ, Carrillo-Reid L, Bargas J (2011) Dopaminergic modulation of striatal neurons, circuits, and assemblies. Neuroscience 198:3-18. doi:10.1016/j.neuroscience.2011.08.051

Svenningsson P, Nishi A, Fisone G, Girault JA, Nairn AC, Greengard $P$ (2004) DARPP-32: an integrator of neurotransmission. Annu Rev Pharmacol Toxicol 44:269-296. doi:10.1146/annurev. pharmtox.44.101802.121415
Ungless MA, Grace AA (2012) Are you or aren't you? Challenges associated with physiologically identifying dopamine neurons. Trends Neurosci 35:422-430. doi:10.1016/j.tins.2012.02.003

Vertes RP (2004) Differential projections of the infralimbic and prelimbic cortex in the rat. Synapse 51:32-58. doi:10.1002/syn.10279

Yamashita N, Hayashi A, Baba J, Sawa A (1997a) Rolipram, a phosphodiesterase-4-selective inhibitor, promotes the survival of cultured rat dopaminergic neurons. Jpn J Pharmacol 75:155-159

Yamashita N, Miyashiro M, Baba J, Sawa A (1997b) Rolipram, a selective inhibitor of phosphodiesterase type 4 , pronouncedly enhanced the forskolin-induced promotion of dopamine biosynthesis in primary cultured rat mesencephalic neurons. Jpn J Pharmacol 75:91-95 